\title{
A Kind of Neither Keynesian Nor Neoclassical Model (4): The Nature of Philips Curve
}

\author{
Zhan Zhan1, Mingan Zhan² \\ ${ }^{1}$ Westa College, Southwest University, Chongqing, China \\ ${ }^{2}$ Yunnan University, Kunming, China \\ Email:1792481782@qq.com
}

How to cite this paper: Zhan, Z. and Zhan, M.G. (2017) A Kind of Neither Keynesian Nor Neoclassical Model (4): The Nature of Philips Curve. Open Access Library Journal, 4: e3388.

https://doi.org/10.4236/oalib.1103388

Received: January 19, 2017

Accepted: March 11, 2017

Published: March 14, 2017

Copyright (c) 2017 by authors and Open Access Library Inc.

This work is licensed under the Creative Commons Attribution International License (CC BY 4.0).

http://creativecommons.org/licenses/by/4.0/

\section{cc) (i) Open Access}

\begin{abstract}
Officials always want to use the replacement relationship between the unemployment rate and the price of the Phillips curve to reduce the unemployment rate, but things go crisscross. The reason is that there is no causal relationship between price and unemployment. In this paper, it demonstrates the Phillips curve is derived from the unemployment cycle equation and the inflation equation. And then, it discusses the influence of core variables and fluctuating variables on Phillips curve and the cause of distortion or complete disappearance of Phillips curve. Finally, this paper discusses monetary neutrality and related monetary policy issues. The policy that is conducive to the smooth operation of the system is consistent with the constant money supply of the economic cycle, rather than counter-cyclical regulation.
\end{abstract}

\section{Subject Areas}

Economics

\section{Keywords}

Philips Curve, Inflation, Unemployment Rate, Monetary Neutral

\section{Introduction}

Although it is very difficult to identify the cause-and-effect relationship between variables, there are many connections between variables in economics. Phillips curve tells us: statistical data of the United Kingdom during 1861-1957 show there is specific substitutional relation between the change rate of nominal wage and unemployment rate [1]. This relation can be explained by the aggregate demand and supply: when the demand curve shift toward left, the equilibrium price, the equilibrium output and employment rate decreases (the unemployment rate increases), the unemployment rate and inflation rate are reverse 
changes.

In this case, both theoretical explanation and statistical data, why does the government not use fiscal policy or monetary policy to increase aggregate demand and reduce the unemployment rate? However, the practice in the United States and Europe in the 1970s shows that the result of this policy has led to higher unemployment. In order to explain this phenomenon, people have built some new ideas, such as inflation expectations, adaptive expectations, etc. [2] [3] [4]. Unfortunately, there are very few people to doubt about the reality of the causal relationship between the unemployment and price in Phillips curve.

\section{The Dominant Phillips Curve}

In the paper "A Kind of Neither Keynesian Nor Neoclassical Model (2): The Business Cycle" [5], we derive the employment cycle equation from the CobbDouglas function: $\dot{R}_{E}=(1-\alpha / \beta) r_{r}+\dot{r}_{r}-\dot{N}$. It shows that:

1) When the change rate of the labor population $\dot{N}$ is constant, the change rate of employment rate $\dot{R}_{E}$ are determined mainly by the real interest rate $r_{r}$.

2) According to the phase diagram $r_{r} \sim \dot{R}_{E}$, we know that the periodicity of $\dot{R}_{E}$ is determined by the periodicity of $r_{r}$. Figure $1(\mathrm{a})$ is the theoretical relationship between the phase diagrams $r_{r} \sim \dot{R}_{E}$ and $r_{r} \sim \dot{r}_{r}$, and Figure 1(b) and Figure 1(c) are their time path diagrams. From the phase diagram $R_{E} \sim \dot{R}_{E}$ in Figure 1(d), we can further draw the time path of the employment rate $R_{E}$, as shown in Figure 1(e).

3) From the relationship between the unemployment rate and the employment rate $R_{U}=1-R_{E}$, we can see that the size and period of $\dot{R}_{U}$ are also determined by $r_{r}$. Figure 1(e) vertical flip, we get Figure 1(f) shows the time path of unemployment rate $R_{U}$.

From the inflation equation $\dot{P}-\theta \ddot{P}=\dot{M}-\dot{Y}_{r} \quad$ [6], we can see that the inflation rate $\dot{P}$ is affected by two factors: the rate of change of money $\dot{M}$ and the rate of change of output $\dot{Y}_{r}$. From the equation of output cycle $\dot{Y}=r+\dot{r}$, we know that the change rate of nominal output is determined by $r$ and its change rate. Although the relationship between $\dot{Y}_{r}$ and $r_{r}$ is more complex than $\dot{Y}$ and $r$, under certain conditions, there is also a real variable equation $\dot{Y}_{r}=r_{r}+\dot{r}_{r}$, which is similar to the nominal variable equation $\dot{Y}=r+\dot{r}$. Therefore, $\dot{P}$ is also affected by the real interest rate $r_{r}$, except for the effect of $\dot{M}$.

Since the cycle of $\dot{M}$ is also determined by the phase diagram $r_{r} \sim \dot{M}$, the cycle of $\dot{P}$ is also determined by $r_{r}$. Figures $1(\mathrm{~g})$-(j) shows briefly the theoretical process. Finally, since the employment rate $R_{E}$ and the inflation rate $\dot{P}$ are in the same direction as the fluctuation of $r_{r}$, the fluctuation of the unemployment rate $R_{U}$ is opposite to the employment rate $R_{E}$, as shown in Figure 1(f). Figure 1(f) and Figure 1(h) show the results of the theoretical derivation: the wave direction of $R_{U}$ is opposite to $\dot{P}$.

Figure 2 is the statistical data of the inflation rate $\dot{P}$ and the unemployment rate $R_{U}$ during 1970-2015 in the United States. In the three periods of 1986-1992, 1998-2002 and 2003-2009 $\dot{P}$ and $R_{U}$ are in accordance with the 

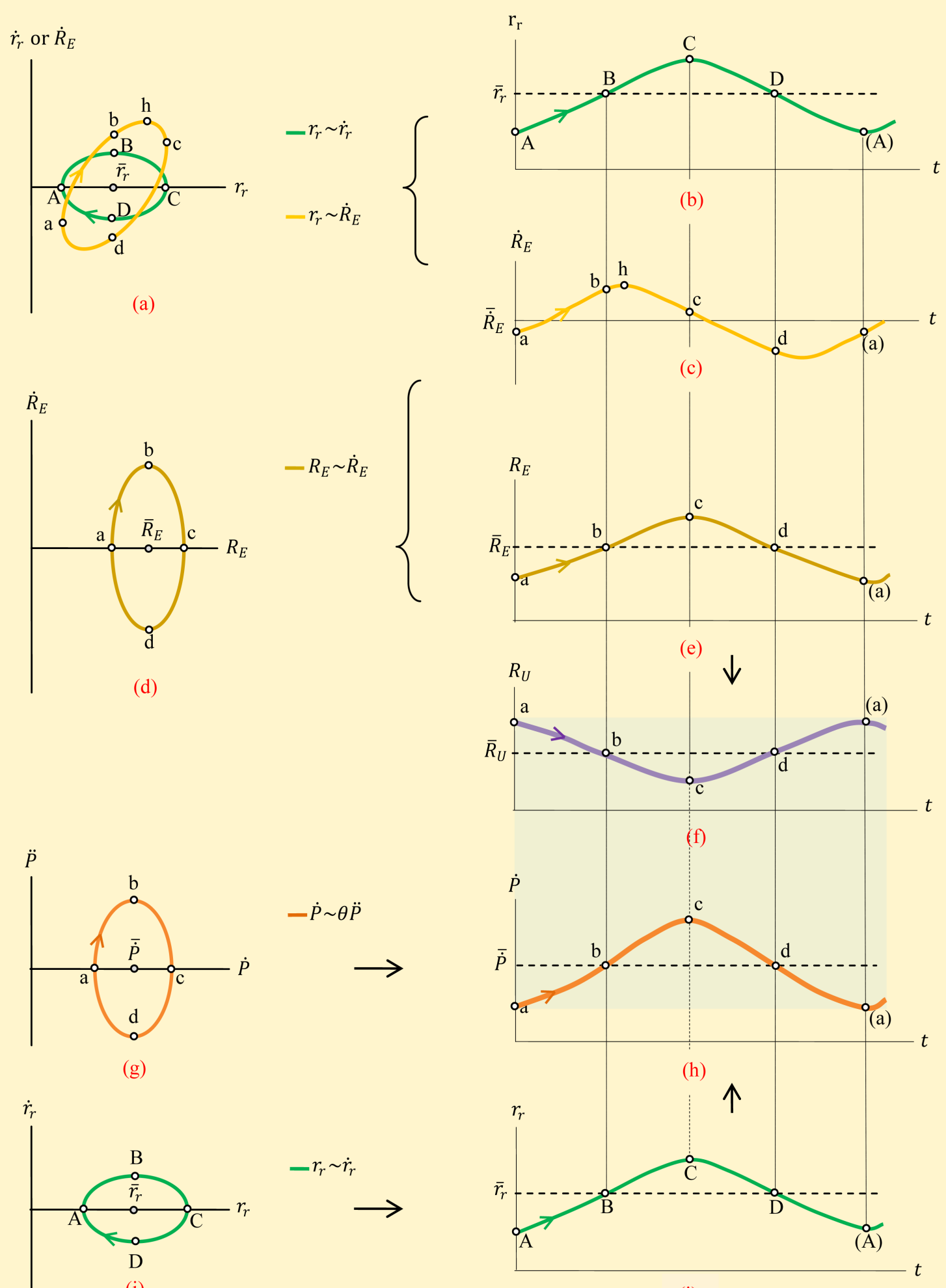

(g)
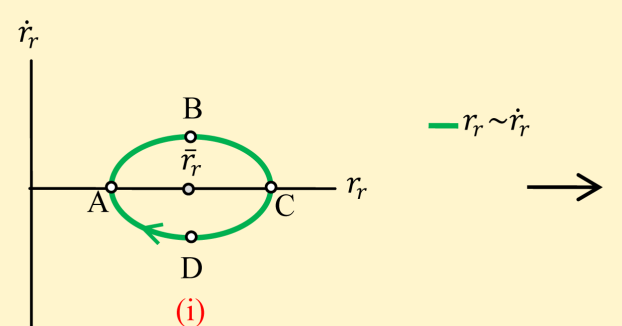

(j)

Figure 1. The reasons of the reverse change of $R_{U}$ and $\dot{P}$ in Phillips curve. Notice: This drawing is from the paper "A kind of neither Keynesian nor neoclassical model (2): The business cycle" [1]. The blue block in the figure warns $R_{U}$ against the direction of $\dot{P}$.

theoretical assumptions of Figure 1. If $R_{U}$ and $\dot{P}$ for the coordinates of drawing with three periods of data, the trend line of the scatter diagram is the 
Phillips curve of the negative slope, as shown in Figure 3.

Looking at the three Phillips curves, we will not be as surprised when A. W. Phillips was at that time. Since we know from the periodic and inflation equations that the decline in $R_{U}$ is not caused by an increase in $\dot{P}$ but by $r_{r}$. In other words, in the Phillips curve, $R_{U}$ and $\dot{P}$ are not causal relations, but they are affected by the same factor and have a correlation.

A further problem is that only three cycles of $R_{U}$ and $\dot{P}$ has Phillips curve characteristics in the nine cycles during 1970-2015. The variables $R_{U}, \dot{P}$ and $r_{r}$ are decomposed into core variables and fluctuating variables,

$R_{U}=\bar{R}_{U}+\tilde{R}_{U}, \dot{P}=\overline{\dot{P}}+\tilde{\dot{P}}$ and $r_{r}=\bar{r}_{r}+\tilde{r}_{r}$, which may help us explain why the Phillips curve disappears. It can be seen from Figure 2 that the Phillips curve appears at a time when the core variable $\overline{\dot{P}}$ is not very high or very low, and the fluctuating variable $\tilde{\dot{P}}$ is not suppressed. This is because $\bar{R}_{U}$ is also stable when $\overline{\dot{P}}$ is relatively stable, and the change of $R_{U}$ is mainly reflected in the periodic variation of $\tilde{R}_{U}$. When $\overline{\dot{P}}$ varies widely, $\overline{\dot{P}}$ may in turn affect $\bar{r}_{r}$,

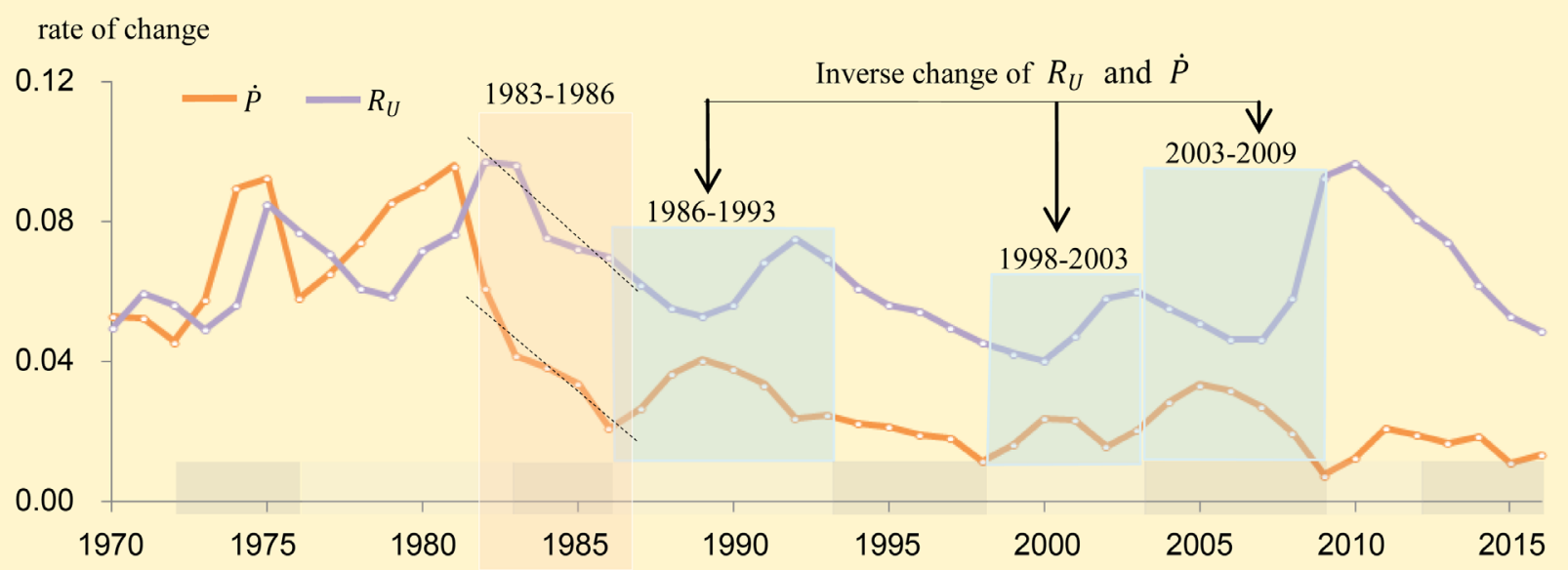

Figure 2. Time Paths of $R_{U}$ and $\dot{P}$ in US statistics. Calculation and resources: 1) $\dot{P}=d P / P \approx \Delta P / P$. The price index $P$ is calculated by deflating the nominal GDP and the real GDP. Data of nominal GDP and real GDP are from http://www.bea.gov. Data of $R_{U}$ are from http://www.bls.gov/.2) The division of the cycle is described in the paper "A kind of neither Keynesian nor neoclassical model (2): The business cycle" [1]. 3) The blue block in the figure warns $R_{U}$ against the direction of $\dot{P}$. 4) The meaning of the two dashed lines in the figure is shown in Figure 5(b).
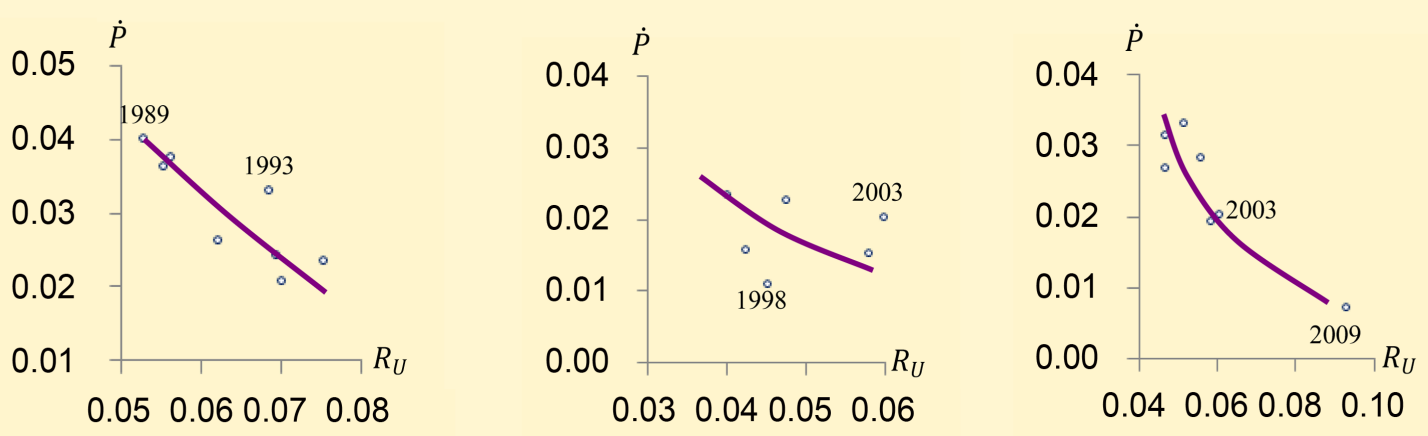

Figure 3. Phillips curves for three periods. Resources: The data of $R_{U}$ and $\dot{P}$ are the same as Figure 2 . 
and the standard form of the Phillips curve will be distorted, or even completely disappear.

\section{The Recessive Phillips Curve}

If the higher core inflation rate $\overline{\dot{P}}$ produces inflation cost, the market efficiency and the core real interest rate $\bar{r}_{r}$ will reduced, and then $\bar{r}_{r}$ will move to the left in Figure 1(a), the core employment rate $\bar{R}_{E}$ will also move to the left in Figure 1(d). Correspondingly, $\bar{r}_{r}$ and $\bar{R}_{E}$ in Figure 1(b) and Figure 1(c) will rotate clockwise and rotate $\bar{R}_{U}$ in Figure 1 (f) counterclockwise. If the higher inflation is managed, $\bar{R}_{U}$ in Figure 1 (f) will rotate clockwise.

Figure 4(a) and Figure 4(b) show respectively the forms of $\dot{P}$ and $R_{U}$ when $\bar{P}$ and $\bar{R}_{U}$ rotate clockwise and counterclockwise. Figure $4(\mathrm{c})$ shows the change forms of $\dot{P}$ and $R_{U}$ when $\bar{P}$ is gradually decreased at the high level. It is shown that the fluctuation variables $\tilde{\dot{P}}$ and $\tilde{R}_{U}$ fluctuate under the influence of $\tilde{r}_{r}$ when the core variables $\overline{\dot{P}}$ and $\bar{R}_{U}$ change, but are often obscured by the changes of the core variables $\bar{P}$ and $\bar{R}_{U}$, especially when the variation of $\overline{\dot{P}}$ and $\bar{R}_{U}$ is large.

In Figure 2, the relationship between $\dot{P}$ and $R_{U}$ is not characteristic of Phillips curve during 1982-1986, and the scatter diagram are shown in Figure 5(a), and the slope of Phillips curve is not negative. However, if the change path of $\dot{P}$

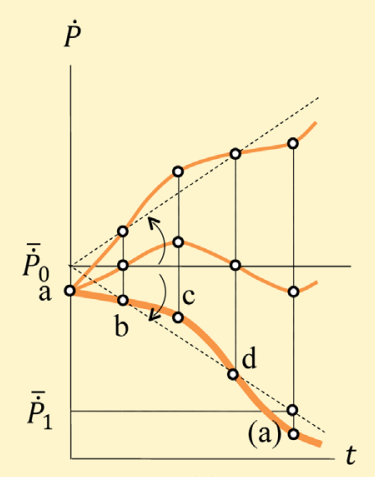

(a)

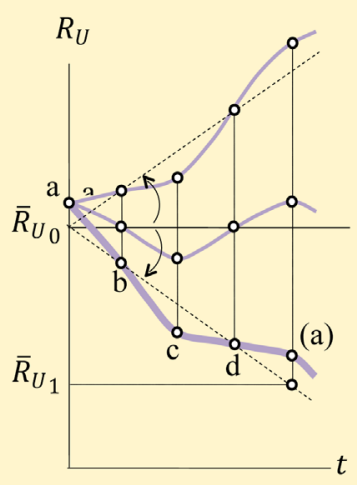

(b)

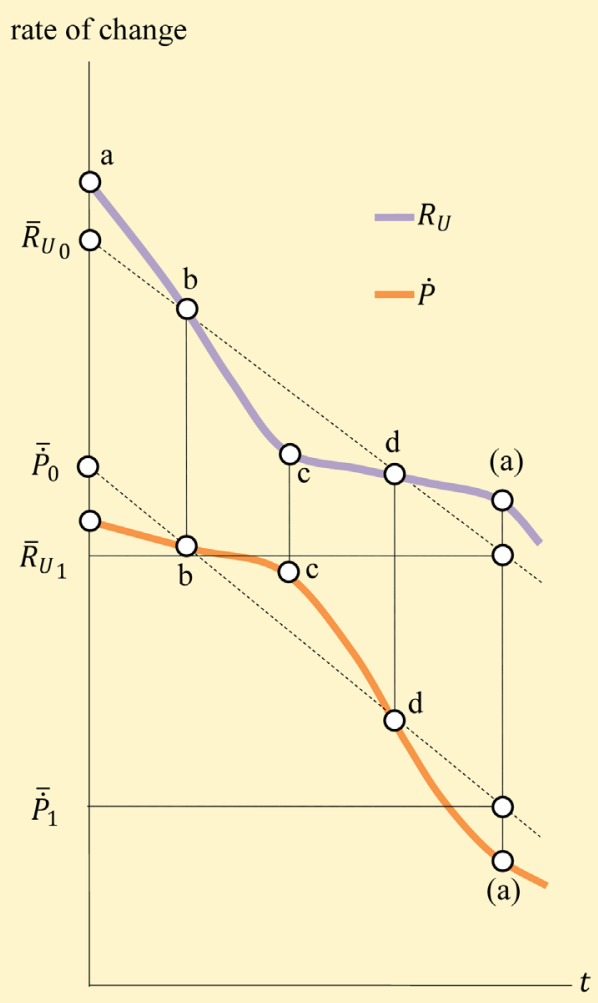

(c)

Figure 4. The time paths of $\dot{P}$ and $R_{U}$ when the change of core variables $\overline{\dot{P}}$ and $\bar{R}_{U}$. Notice: Figure $4(\mathrm{c})$ shows the case when $\bar{P}$ and $\bar{R}_{U}$ are continuously decreasing. 


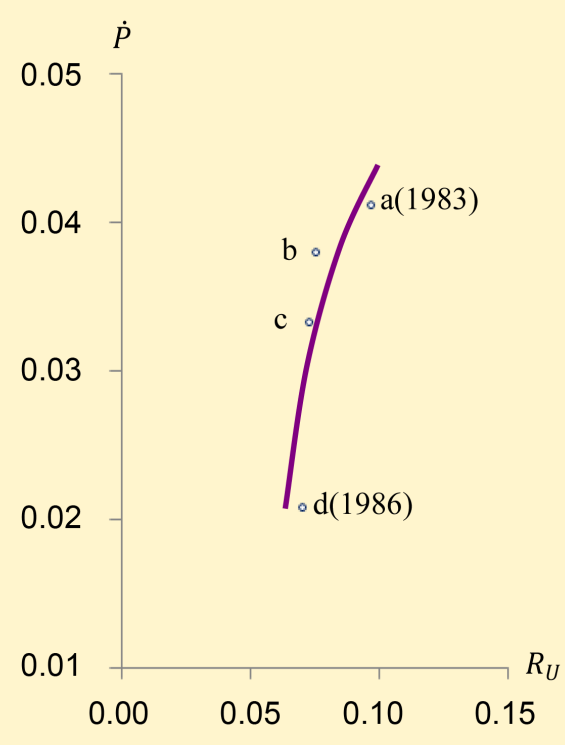

(a)

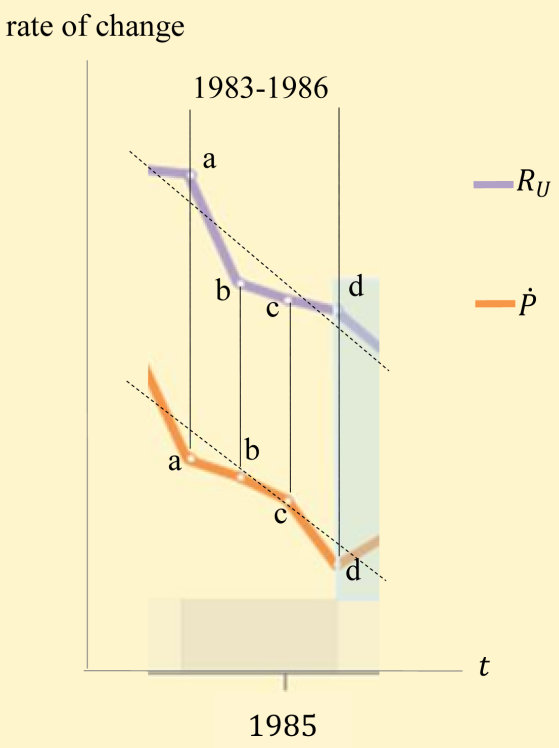

(b)

Figure 5. (a) Phillips curve during 1983-1986. The data sources of $R_{U}$ and $\dot{P}$ are the same as Figure 2. Figure 5(b) The time path of $R_{U}$ and $\dot{P}$ during 1983-1986 copy from part of Figure 2.

and $R_{U}$ in the period of 1982-1986 is cut off as shown in Figure 5(b), it will be very similar to the prediction of Figure 4 (c). In the cycle during 1982-1986, $\dot{P}$ and $R_{U}$ still have the characteristics of the reverse fluctuations, but because $\bar{P}$ and $\bar{R}_{U}$ change in a larger range to cover the changes of $\tilde{\dot{P}}$ and $\tilde{R}_{U}$, so that $\dot{P}$ and $R_{U}$ become the recessive Phillips curve.

This shows that the reversal of the relationship between $R_{U}$ and $\dot{P}$, which was discovered by A. W. Phillips in 1958, is an inverse change of $\tilde{\dot{P}}$ and $\tilde{R}_{U}$ in the business cycle. The change of $\bar{P}$ and $\bar{R}_{U}$ may obscure the change of $\tilde{\dot{P}}$ and $\tilde{R}_{U}$ when the changes of $\bar{P}$ and $\bar{R}_{U}$ are large, so that the reverse change between $R_{U}$ and $\dot{P}$ disappears.

Although the dominant Phillips curve will disappear when the change of $\bar{P}$ and $\bar{R}_{U}$ is large, the Recessive Phillips curve still exists in most cases, because $\tilde{\dot{P}}$ and $\tilde{R}_{U}$ are still in the opposite direction. This can be used to filter out the trend changes in $R_{U}$ and $\dot{P}$, by means of differential methods and to keep the fluctuations in $R_{U}$ and $\dot{P}$, that is, to observe the fluctuations of $\dot{R}_{U}$ and $\ddot{P}$.

As shown in Figure 6, in the nine cycles from 1970 to 2015, except for two cycles of 1972-1976 and 1993-1998, the fluctuation directions of $\dot{R}_{U}$ and $\ddot{P}$ are obviously opposite. This indicates that the variation of $R_{U}$ and $\dot{P}$ in these seven cycles is not the dominant or recessive Phillips curve.

In the 1970s, as Herbert Stein said, "It was a messy time". when President Richard Nixon was in power [7]. Although $\tilde{R}_{U}$ and $\tilde{\dot{P}}$ are still affected by $\tilde{r}_{r}$, the rapid rise of $\bar{P}$ leads directly to the decrease of $\bar{r}_{r}$, so before $1976, R_{U}$ and $\dot{P}$ are rapidly increased by $\bar{P}$ and $\bar{R}_{U}$ Kidnapping, the stagflation has become the economic characteristics of the time. In higher inflation, the cyclical 


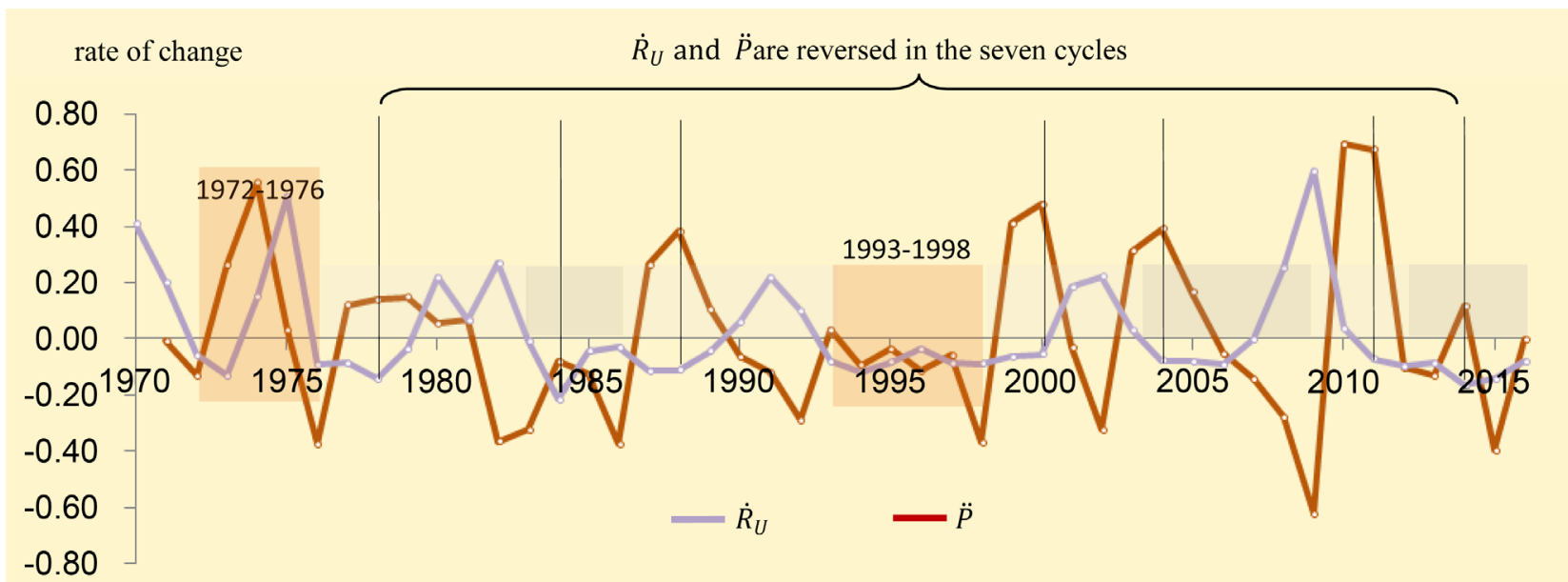

Figure 6. Comparison of the fluctuations of $\dot{R}_{U}$ and $\ddot{P}$. Calculation and resources: 1) $\dot{R}_{U}=d R_{U} / R_{U} \approx \Delta R_{U} / R_{U}$, $\ddot{P}=d \dot{P} / \dot{P} \approx \Delta \dot{P} / \dot{P}$. The data of $R_{U}$ and $\dot{P}$ are the same as Figure 2. 2) The division of the cycle is the same as Figure 2. The red block indicates that $\dot{R}_{U}$ and $\ddot{P}$ are not in the opposite direction (neither dominant nor recessive Phillips curves).

relationship between $\dot{P}$ and other variables may disappear. Especially in the case of hyperinflation, the change of $\tilde{\dot{P}}$ is small enough to be negligible compared to the large change of $\overline{\dot{P}}$.

The 1990s were times when Federal Reserve Chairman Alan Greenspan used monetary policy to fine-tune the discount rate, inflation, and unemployment. It can be seen from Figure 2 that in the period of 1992-1998, the fluctuation of $R_{U}$ and $\dot{P}$ is almost completely controlled, and the decline of $R_{U}$ is more obvious than $\dot{P}$. This, on the other hand, distorts the standard Phillips curve: inflation and unemployment may both rise and decline in the same direction, and the latter change is the ideal state in which people dream. However, this attempt to eliminate the precise control of the Phillips curve became the important cause of the subsequent financial tsunami.

The above analysis shows that the variation of the core variables $\bar{P}$ and $\bar{r}_{r}$ is distinguished from the variation of the fluctuation variables $\tilde{\dot{P}}$ and $\tilde{r}_{r}$ to explain more clearly why $\dot{P}$ and $R_{U}$ are sometimes reverse-changing and sometimes changing in the same direction.

Whether the dominant or recessive Phillips curve, from the previous analysis can be drawn the following conclusions:

1) The relationship between $\dot{P}$ and $R_{U}$ in the Phillips curve is not causal.

2) The fluctuation of $\tilde{r}_{r}$ is the common cause of the fluctuations of $\tilde{\dot{P}}$ and $\tilde{R}_{U}$.

3) When the variation range of $\bar{P}$ is large, the change of $\bar{P}$ will not only obscure the change of $\tilde{\dot{P}}$, but also cause $\bar{r}_{r}$ to decrease, though it will not occur in the current period.

4) The dominant Phillips curve appears at the time when $\overline{\dot{P}}$ is relatively stable and $\tilde{\dot{P}}$ is not suppressed.

\section{Conditions of Monetary Neutrality}

The analysis of the implicit Phillips curve gives us a deep understanding of the 
relationship between inflation and unemployment, but it still fails to answer the most important practical problem in monetary research: "Is money only affecting prices, or both price and real output?" Or under what circumstances money is neutral and what is non-neutral. From the simplest quantity equation $a M=P Y_{r}$, to the quantity equation $\dot{P}-\theta \ddot{P}=\dot{M}-\dot{Y}_{r}$ which reflects the cyclicality of money, we still cannot answer this question. Even if the US dollar against the Japanese Yen drops from 1 USD/120 JPY to 1 USD/1 JPY, only the exchange coefficient $a$ in the quantity equation $a M=P Y_{r}$ needs to be adjusted, it's still not clear what effects that $M$ will put on $Y_{r}$. Since $Y_{r}$ is determined by the Cobb-Douglas function and not by the money-quantity equation, our macroeconomic variable equation cannot answer this question. This is a microeconomic problem.

As the credit card payment and Internet payment is not popular in India, the abolition of two current banknotes on November 8, 2016 has caused problems of market transactions. Therefore the international credit rating company lowered the 2016 economic growth rate of India. In the case of a decline in production efficiency due to a shortage of cash, supplementing or increasing the money supply is certainly conducive to the recovery of GDP. But if it was not the case that the lack of banknotes causes the increase of transaction cost, such as in India, would the increase of money supply bring up the real output?

In addition to the dominant function as a trading media, wealth storage and measurement of wealth unit, money also has a function of transferring wealth that is easily to be neglected. For example, the actual wealth of creditors gradually reduced in a certain monetary policy, or the burden of the debtor continues to increase under a certain monetary policy. If the expansion of the central bank balance sheet could raise GDP, it is certainly not a direct process as the increase of savings is the increase of real wealth in personal accounts. It should be the wealth transfer effect of money supply changes the operating efficiency of economic systems, and then cause a change in GDP. In the microeconomic market, any transfer or movement of wealth needs to be related to production or service, and because the process is completed in competition, it is efficient. Does the wealth transfer effect caused by the increase or decrease of money supply is efficient?

From the perspective of transfer payments, the role of monetary and fiscal policies is similar. Fiscal policy is the formation of transfer payments between taxpayers and governments or between different industries by increasing taxes or bonds. Monetary policy is a transfer between debtors and creditors.

As a social responsibility, the government sometimes has to do something, but it is not as Keynesian theory describes that government spending will have a multiplier effect. The most extreme case of fiscal policy is that when the national tax or debt is not sufficient to maintain the liquidity of finance, the spending of government can only rely partly or entirely on the increase issuance of money. This appears to be a fiscal act, but essentially it is a monetary policy abducted by fiscal policy. It has a greater impact on the operational efficiency of the system 
than fiscal or monetary policy alone, since these "Seigniorage" may have no connection with production and services.

The downside of inflation is not the negligible transaction costs such as "shoe-leather cost" or "menu cost", but the inefficient or low-efficient production caused by the increase or decrease in wealth that does not correspond to production or services. On the one hand, companies with higher production costs or poor market demand may not be eliminated because of the debt burden under inflation. On the other hand, during the deflation, the money savers can gain the rewards of wealth without competition under deflation.

Therefore, though we cannot find the effect of $\dot{M}$ on $\dot{Y}_{r}$ in the equation $\dot{P}-\theta \ddot{P}=\dot{M}-\dot{Y}_{r}$, the wealth transfer effect caused by the change of money supply would affect $\dot{Y}_{r}$ by changing $\dot{P} . \dot{Y}_{r}$ is decided by its marginal state $r_{r}$, and since $r_{r}$ is more sensitive than $\dot{Y}_{r}$ in reacting to $\dot{M}$, we choose to observe the variation trend of $\dot{M}$ and $r_{r}$ in statistical data.

Figure 7 shows that during the period of stagflation in 1970-1976, $\overline{\dot{M}}$ increased, $\bar{r}_{r}$ decreased and the value was negative in 1974-1975. It indicates that the expansion of the currency severely damaged the operating efficiency of the system. During 1976-1989, the declining trend of $\overline{\dot{M}}$ and the self-repair capacity of market led to a sharp rise in $\bar{r}_{r}$, and then $\bar{r}_{r}$ stabilized at a high level of around 0.04 . The highly praised Reagan economy actually should credit President Carter's control of inflation and fiscal spending that began in the late 1970s [7].

$\bar{r}_{r}$ began to decline in 2001, and in 2011-2014 its value was less than 0 . The reason is due to the impact of low $\overline{\dot{M}}$ during 1990-1995, and more importantly, the interest rate dependent syndrome (IRDS) for interest rate controls created by Federal Reserve Chairman Alan Greenspan and his successor. The financial crisis in 2008, in fact, is the result of the economic system adjusted itself since it could not bear with people always benefitted through the housing finance under

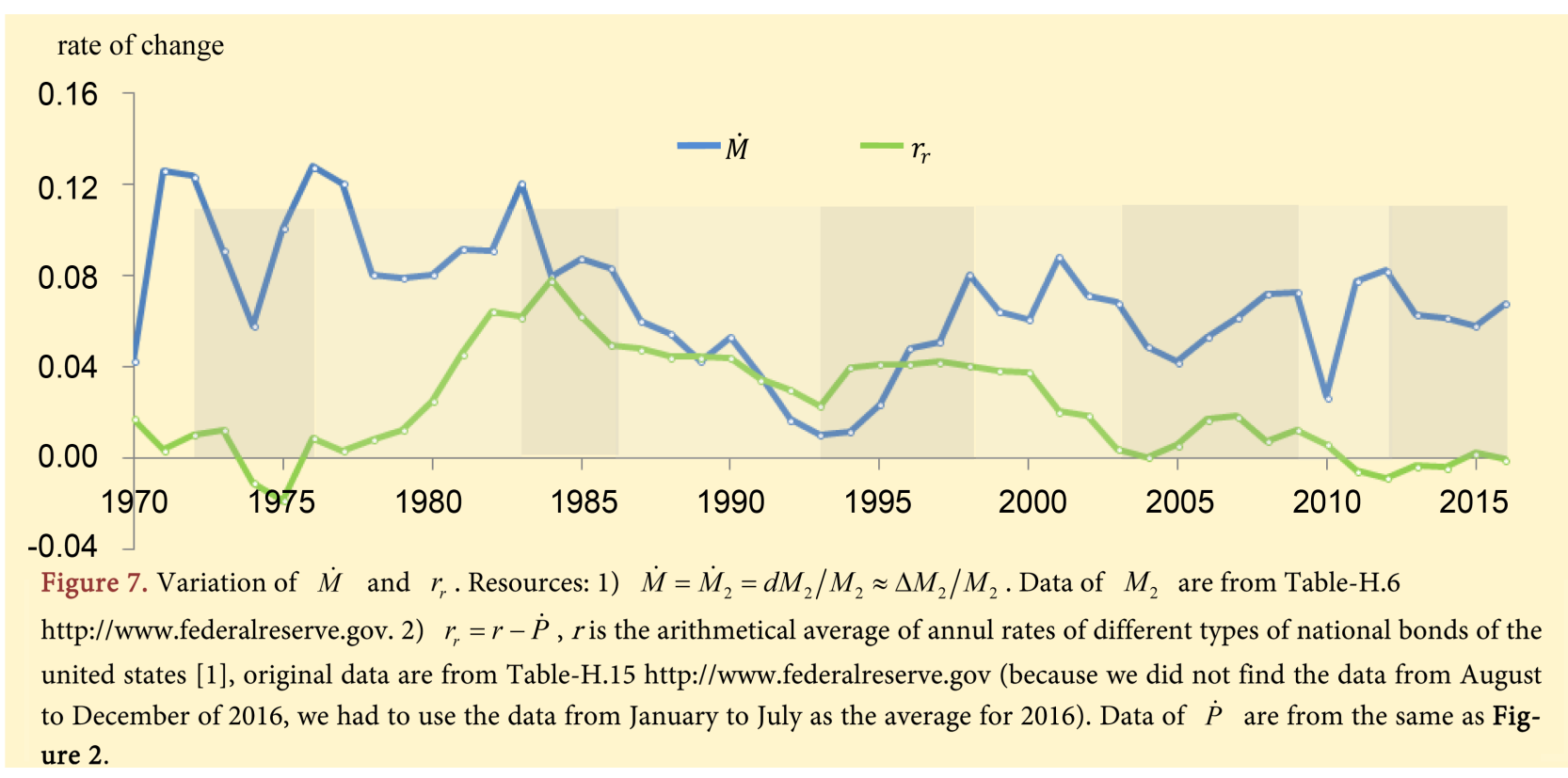


the low interest rate policy.

Therefore, according to the statistics of $\dot{M}$ and $r_{r}$, the effect of varying money on the central bank's balance sheet is not neutral. In the field of microeconomics, the occasional transfer of wealth (such as donations of heritage) that does not correspond to the production or services has limited effects on the efficiency of the entire economic system. However, inflation or deflation in the macroeconomics field will have a negligible impact on the system.

The non-neutrality of money is not only reflected in the effect of transfer payments, but also on the consistency of the relationship between the actual variables and the nominal variables, that is, the "coordination effect" of money.

Before the appearance of periodic equation $\dot{Y}=r+\dot{r}$, we can directly convert actual values by the definition between actual values and nominal values. For example: the actual output and nominal output can be converted according to the equation $Y_{r}=Y / P$ or $\dot{Y}_{r}=\dot{Y}-\dot{P}$; the basic equation of nominal variables $\Delta Y=r Y$ [8], can be directly rewritten as the basic equation of actual variables $\Delta Y_{r}=r_{r} Y_{r}$. However, considering the periodic relationship between variables, the nominal variable cannot be directly rewritten as the actual variables in some equations. For example, $\dot{Y}=r+\dot{r}$ cannot be rewritten as $\dot{Y}_{r}=r_{r}+\dot{r}_{r}$.

In general, only when $\dot{P}=0$ or $\dot{P}=r_{r}$, there is no difference between the actual variables and the nominal variables in the macroeconomics system. From $r=r_{r}+\dot{P}$,

$$
\dot{Y}=r+\dot{r}=r_{r}+\dot{P}+\frac{d\left(r_{r}+\dot{P}\right)}{r_{r}+\dot{P}}=r_{r}+\dot{P}+\frac{r_{r}}{r_{r}+\dot{P}} \cdot \frac{d r_{r}}{r_{r}}+\frac{\dot{P}}{r_{r}+\dot{P}} \cdot \frac{d \dot{P}}{\dot{P}},
$$

substitute the left side of the above equation with $\dot{Y}=\dot{Y}_{r}+\dot{P}$, then,

$$
\dot{Y}_{r}=r_{r}+\frac{r_{r}}{r_{r}+\dot{P}} \dot{r}_{r}+\frac{\dot{P}}{r_{r}+\dot{P}} \ddot{P}
$$

When $\dot{P}=0$, the above equation can be simplified as $\dot{Y}_{r}=r_{r}+\dot{r}_{r}$. And when $\dot{P}=r_{r}$, since

$$
\ddot{P}=\frac{d \dot{P}}{\dot{P}}=\frac{d r_{r}}{r_{r}}=\dot{r}_{r}
$$

then,

$$
\dot{Y}_{r}=r_{r}+\frac{r_{r}}{r_{r}+\dot{P}} \dot{r}_{r}+\frac{\dot{P}}{r_{r}+\dot{P}} \ddot{P}=r_{r}+\frac{r_{r}}{r_{r}+\dot{P}} \dot{r}_{r}+\frac{r_{r}}{r_{r}+r_{r}} \dot{r}_{r}=r_{r}+\dot{r}_{r}
$$

This is the reason that the relation of $\dot{Y}_{r}$ and $r_{r}$ can only be expressed as $\dot{Y}_{r} \approx r_{r}+\dot{r}_{r}$ in our previous paper, and the fluctuation of actual variables is different from and even more volatile than that of nominal variables.

Since the difference of fluctuation between actual and nominal variables may lead to more speculative activities and then lower the efficiency of market system operation. Therefore the better the consistency of actual and nominal variables, and the closer the fluctuation pattern is in the time path, the smaller the loss of system operating efficiency. To study the so-called monetary neutrality, we not only need to consider the transfer payment when price changes, but also the 
coordination effect between actual and nominal variables.

It is very difficult to make $\dot{P}=0$ because of the change of $\dot{P}$ and $\tilde{\dot{P}}$. Theoretically, the central bank can offset the change in $\tilde{\dot{P}}$ by controlling $r_{r}$ or $\overline{\dot{M}}$ (as the Federal Reserve did in the cycle of 1992-1998) to achieve this goal. However, since achieving this goal will suppress the natural fluctuation of the system and then cause problems to the coordination between actual and nominal variables, the choice of taking accounts of both sides is to let the inflation rate equal the real interest rate, namely $\dot{P}=r_{r}$.

Friedman used to think that the US dollar could grow at a constant rate of 3\% 5\% per year [1]. According to the average value 0.0229 of $r_{r}$ from 1970 to 2016, according to the coordination effect of $\dot{P}=r_{r}, \quad \bar{M}=2 \times 0.0229=0.0458$. This is within the scope of Friedman's proposal. If this is done, the average of the inflation rate will be $\bar{P}=\bar{r}_{r}=0.0229$. This kind of inflation rate that doubles the real interest rate should be moderate inflation. Experience tells us that moderate inflation will not cause damages to economic operating efficiency. This is because under stable money supply, the capital market will develop financial products that can reduce or even eliminate the effect of transfer payments.

Because of the adaptability and offsetting effects of the capital market, the effect of monetary changes on $r_{r}$ appears to be in terms of cycles, so that even under "the constant currency rule", several cycles of adaptive adjustment are required before the system approaches the so-called ideal operating state. In this state, various variables will continue to fluctuate, but their trend line will be close to a horizontal line, the phase difference of fluctuations will be more consistent with our theoretical analysis in the periodic equation. Figure 8 is a schematic view of this ideal state.

Monetary policy in traditional macroeconomics has two important roles: first, to ensure the smooth operation of the economy, and second, to promote economic growth. Fluctuations of variables from 1970 to 2015, except the correctional

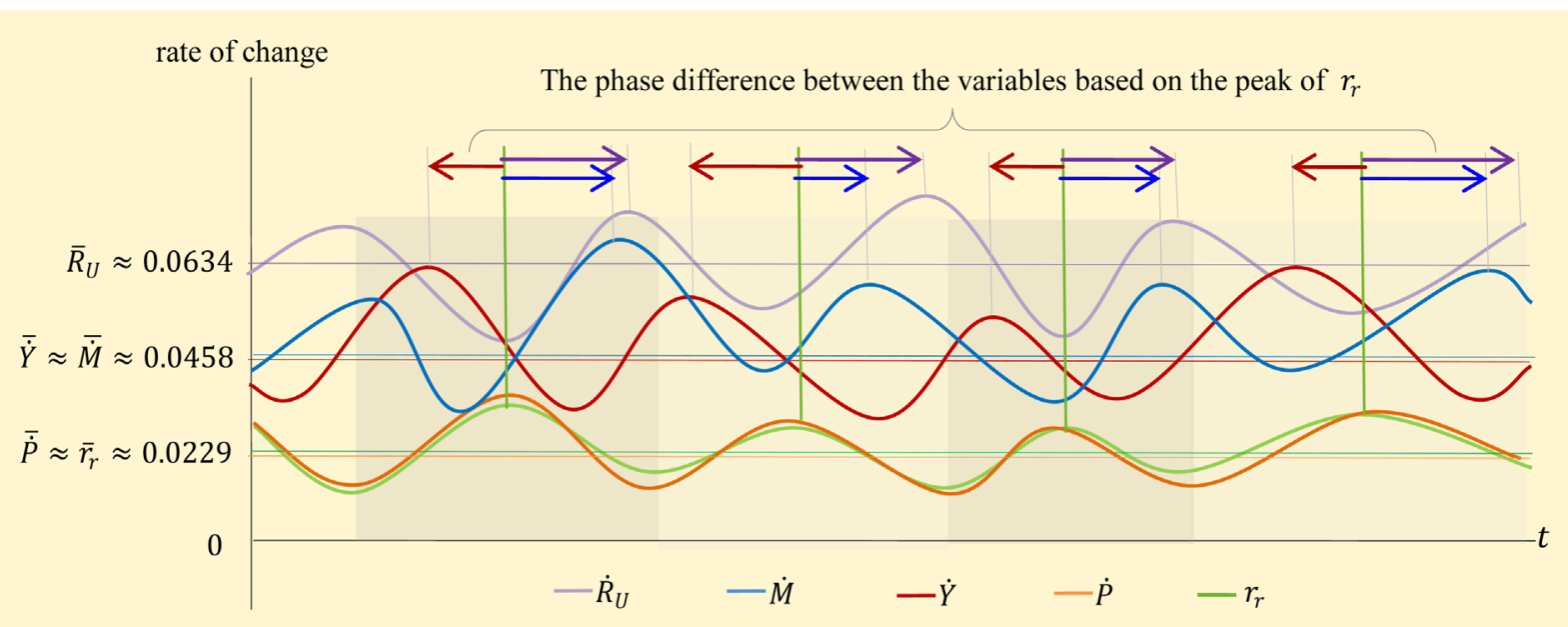

Figure 8. The variation trend of each variable and the phase difference of fluctuation under the ideal state. Note: The light and dark stripes in the figure are assumed to be four cycles, and the dotted line is the trend line of each variable. The number of trend lines on the ordinate is the estimated value from the average of variables in 1970-2016. 
monetary policy in Reagan period, it is difficult to say that the US Federal Reserve's monetary policy has played these two roles. For the first role, we theoretically have proved that there is no fluctuation only under the simple reproduction state that the marginal output equals to 0 . The real smooth operation is not to smooth the economic cycle, but to make the relationship between variables in the fluctuation simple and clear. Countercyclical monetary policy will not only weaken the market vitality, and even cause economic crisis. The second role is usually to stimulate the economy with money. If printing banknotes could make net increases of real wealth grow, even it would multiply the inflation rate is also cost-effective, because the cost of printing banknotes is negligible compared with manufacturing cars and building houses not to mention in modern technology we can use electronic money without printing costs.

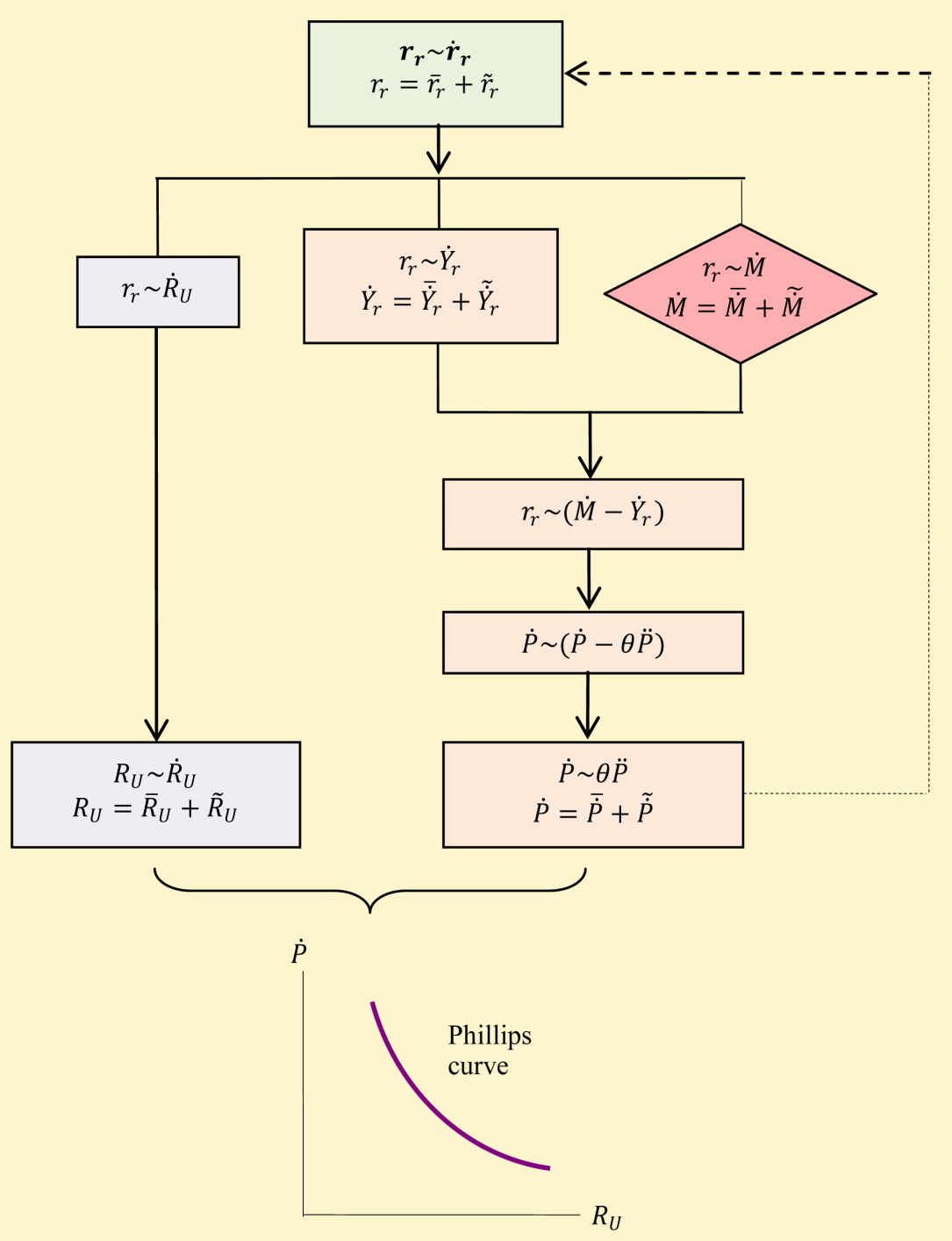

Figure 9. The principle of forming the Phillips curve. The meanings of symbols in the figure are described in references [5] [6]. 
The business cycle is one way of keeping the system alive and does not conflict with the microeconomic mechanisms. Just as four seasons can promote plant metabolism: prosperity is the spring of output growth, but also the hot bed for inefficient producers and production projects; only those enterprises that are able to survive in the winter of recession will make a real contribution to the production efficiency of the system. Therefore, the endogenous cyclical fluctuation in the system is not our enemies, but economics policies that tell us currency are actual wealth and use currency to against the business cycle.

\section{Conclusions}

For a fact without supporting from theories, it is difficult to say that this fact is a general law; similarly, for a theory without approving from fact, it is difficult to be sure of this theory. According to our theory of economic cycle and inflation, the form of Phillips curve shows in Figure 9. In the Phillips curve, there is no causal relationship between the inflation rate and the unemployment rate, and they appear to be linked because they are all affected by changing real interest rate.

For the question "does the state of money only affect the price or both price and real output?", the analysis of this paper shows that the change of money supply would affect both price and real output in the short run, but the effect is normally negative, since the transfer payment that is not linked to any production and service would affect the operating efficiency of the system. The idealist state of money should be that people could use them to accomplish tempted trade, and the distribution of actual wealth would not be affected by the amount of money change.

\section{References}

[1] Phillips, A.W. (1958) The Relation between Unemployment and the Rate of Change of Money Wage Rates in the United Kingdom, 1861-1957. Economica, 25, 283-299. https://doi.org/10.1111/j.1468-0335.1958.tb00003.x

[2] Friedman, M. (1968) The Role of Monetary Policy. American Economic Review, 58, $1-17$.

[3] Friedman, M. (1977) Nobel Lecture: Inflation and Unemployment. Journal of Political Economy, 85, 451-472. https://doi.org/10.1086/260579

[4] Phelps, E. (1967) Phillips Curves, Expectation, and Optimal Inflation over Time. Economica, 135, 254-281. https://doi.org/10.2307/2552025

[5] Zhan, M.A. and Zhan, Z. (2016) A Kind of Neither Keynesian Nor Neoclassical Model (2): The Business Cycle. Open Access Library Journal, 3, e3215. https://doi.org/10.4236/oalib.1103215

[6] Zhan, M.A. and Zhan, Z. (2017) A Kind of Neither Keynesian Nor Neoclassical Model (3): The Inflation Equation. Open Access Library Journal, 4, e3333.

[7] Herbert, S. (1994) Presidential Economics: The Making of Economic Policy from Roosevelt to Clinton. 3rd Revised Edition, American Enterprise Institute for Public Policy Research, Washington DC.

[8] Zhan, M.A. and Zhan, Z. (2016) A Kind of Neither Keynesian Nor Neoclassical 
Model (1): The Fundamental Equation. Open Access Library Journal, 3, e3207. https://doi.org/10.4236/oalib.1103207

Submit or recommend next manuscript to OALib Journal and we will provide best service for you:

- Publication frequency: Monthly

- 9 subject areas of science, technology and medicine

- Fair and rigorous peer-review system

- Fast publication process

- Article promotion in various social networking sites (LinkedIn, Facebook, Twitter, etc.)

- Maximum dissemination of your research work

Submit Your Paper Online: Click Here to Submit

Or Contact service@oalib.com 